\title{
STRUCTURE OF THE FLOW AND YAMADA POLYNOMIALS OF CUBIC GRAPHS
}

\author{
IAN AGOL AND VYACHESLAV KRUSHKAL
}

\begin{abstract}
We establish a quadratic identity for the Yamada polynomial of ribbon cubic graphs in $\mathbb{R}^{3}$, extending the Tutte golden identity for planar cubic graphs. An application is given to the structure of the flow polynomial of cubic graphs at zero. The golden identity for the flow polynomial is conjectured to characterize planarity of cubic graphs, and we prove this conjecture for a certain infinite family of non-planar graphs.

Further, we establish exponential growth of the number of chromatic polynomials of planar triangulations, answering a question of D. Treumann and E. Zaslow. The structure underlying these results is the chromatic algebra, and more generally the $\mathrm{SO}(3)$ topological quantum field theory.
\end{abstract}

\section{INTRODUCTION}

Using the interplay between classical and quantum polynomials of graphs and ideas from topological quantum field theory (TQFT), we establish results on the structure of the Yamada and flow polynomials of cubic graphs. It has been known since the work of Birkhoff and Lewis in the 1940s [3] that the values $(3 \pm \sqrt{5}) / 2$ of the parameter play a special role in the theory of the chromatic polynomial $\chi_{T}$ of planar triangulations. In a series of papers $[19,20]$ in the 1960s, Tutte established further remarkable properties, including the golden identity. Formulated dually in terms of the flow polynomial $F_{G}$ of planar cubic graphs $G$, it reads

$$
F_{G}(\phi+2)=\phi^{E} F_{G}(\phi+1)^{2},
$$

where $E$ is the number of edges of $G$ and $\phi$ denotes the golden ratio $(1+\sqrt{5}) / 2$. The special role played by $\phi+1$, and more generally by the Beraha numbers [2] $B_{n}=2+2 \cos (2 \pi / n)$, was conceptually explained in [6] where these results were placed in the context of $\mathrm{SO}(3) \mathrm{TQFT}$.

We show in Theorem 3.1 that Tutte's identity (1.1) admits an extension to the Yamada polynomial $R_{G}$ of ribbon cubic graphs $G$ in $\mathbb{R}^{3}$ :

$$
R_{G}\left(e^{\pi i / 5}\right)=(-1)^{V-E} \phi^{E} R_{G}\left(e^{-2 \pi i / 5}\right)^{2},
$$

where $V, E$ denote the number of vertices and edges of $G$, respectively. In fact, (1.2) is a common extension of (1.1) and of the identity for links, relating the 2-colored 
Jones polynomial at $e^{\pi i / 10}$ and the square of the Jones polynomial at $e^{-\pi i / 5}[13$, Corollary 4.16], see section 3.

The Yamada polynomial [24] is a quantum invariant of ribbon graphs [15] in $\mathbb{R}^{3}$, corresponding to the adjoint representation of $U_{q}\left(\mathfrak{s o}_{3}\right)$. Conceptually, as discussed in [6, Section 5] and also [13, Section 4.4], the reasons underlying the golden identity are the level-rank duality between the $\mathrm{SO}(3)_{4}$ and the $\mathrm{SO}(4)_{3}$ TQFTs, and the isomorphism $\mathfrak{s o}(4) \cong \mathfrak{s o}(3) \times \mathfrak{s o}(3)$.

Concretely, the Yamada polynomial is defined by the contraction-deletion rule and the $S O(3)$ Kauffman skein relation, see section 2.1 for details. For planar graphs $G$, the Yamada polynomial coincides with a renormalization of the flow polynomial:

$$
F_{G}(Q)=(-1)^{V-E} R_{G}(q), \text { where } Q=q+2+q^{-1} .
$$

For non-planar graphs (and for knotted embeddings of planar graphs) the Yamada polynomial carries a lot of information about the embedding of a ribbon graph in 3 -space, and so in general the Yamada polynomial of a ribbon graph and the flow polynomial of the underlying abstract graph are quite different.

In contrast with (1.1), in [1] we formulated a conjecture that the Tutte golden identity for the flow polynomial characterizes planarity of cubic graphs:

Conjecture 1.1. For any cubic bridgeless graph $G$,

$$
(-\phi)^{E} F_{G}((5-\sqrt{5}) / 2) \geq F_{G}((3-\sqrt{5}) / 2)^{2},
$$

Moreover, $G$ is planar if and only if (1.3) is an equality.

The inequality at the Galois conjugate values,

$$
F_{G}(\phi+2) \leq \phi^{E} F_{G}(\phi+1)^{2},
$$

conjecturally also holds for any cubic graph $G$, with an equality if and only if $G$ is planar. In section 5 we prove the conjecture for a family of near-planar graphs (which have a planar projection with a single crossing); Conjecture 1.1 in general remains open.

It is interesting to note that as a consequence of (1.1), there is a relation between the values of the flow polynomial of planar cubic graphs at 0 and 4 :

$$
F_{G}(0) \equiv 3^{E} F_{G}(4)^{2}(\bmod 5),
$$

see lemma 4.1. More generally, a congruence $(\bmod 5)$ between the values $R_{G}(-1)$, $R_{G}(1)$ implies an extension of (1.5) to all cubic graphs,

$$
F_{G}(0) \equiv 3^{E} R_{G}(1)^{2}(\bmod 5),
$$

where the value $R_{G}(1)$ is also known as the Penrose number of $G$ (defined up to a sign), see section 4 . Using these relations, we give an application to the structure $(\bmod 5)$ of the flow polynomial at zero for cubic graphs. This value is known [4] to count Eulerian equivalence classes of totally cyclic orientations. 
Theorem 1.2. Let $G$ be a cubic graph with $V$ vertices. Then the value of the flow polynomial of $G$ at zero satisfies:

$$
\begin{gathered}
F_{G}(0) \equiv 0,1,4(\bmod 5) \text { if } V / 2 \text { is even, } \\
F_{G}(0) \equiv 0,2,3(\bmod 5) \text { if } V / 2 \text { is odd. }
\end{gathered}
$$

Moreover, suppose $G$ is a snark, that is a bridgeless cubic graph with chromatic index 4. Then $F_{G}(0) \equiv 0(\bmod 5)$, and therefore $F_{G}(0)$ is divisible by 120 .

The proofs of the results in this paper are given in the context of the chromatic algebra [6], and more generally the $\mathrm{SO}(3)$ TQFT. Using these methods, we also answer a question of D. Treumann and E. Zaslow [16] about the asymptotics of the number of chromatic polynomials of planar triangulations, motivated by their work on Legendrian surfaces [17].

Theorem 1.3. The number of chromatic polynomials of planar triangulations with $n$ vertices grows exponentially in $n$.

An exponential upper bound is well-known, and it can be deduced from Tutte's enumeration of planar triangulations [18]. We show that the chromatic algebra contains a free semigroup, yielding an exponential lower bound, see section 6. Conceptually, this may be viewed as an application of the Tits alternative for semigroups.

The key ingredients used throughout the paper - the chromatic algebra and the flow category - are recalled in section 2. The identity (1.2) for the Yamada polynomial is established in section 3. Section 4 discusses the structure of the flow polynomial at zero $(\bmod 5)$ and gives a proof of theorem 1.2 . The proof of conjecture 5.1 for a collection of near-planar graphs is given in section 5. Since the original Tutte golden identity (1.1) serves as the motivation for several results in this paper, for convenience of the reader we include its proof in section 7 .

\section{Graph polynomials, Algebras And CATEGories}

This section summarizes the relevant background material and notation used in the paper.

2.1. The chromatic and flow polynomials. The flow polynomial $F_{G}(Q)$ of a graph $G$ satisfies the contraction-deletion rule: given an edge $e$ of $G$ which is not a bridge,

$$
F_{G}(Q)=F_{G / e}(Q)-F_{G \backslash e}(Q) .
$$

If $G$ contains a bridge, $F_{G} \equiv 0$. The flow polynomial of a graph consisting of a single vertex and $n$ loops is defined to be $Q^{n}$, and the polynomial is multiplicative with respect to taking the disjoint union. 
For planar graphs $G$, the flow polynomial is essentially the chromatic polynomial $\chi_{G^{*}}$ of the dual graph $G^{*}$ :

$$
F_{G}(Q)=Q^{-c} \chi_{G^{*}}(Q),
$$

where $c$ is the number of connected components of $G$. The flow polynomial was defined by Tutte [21], and in fact both the chromatic and flow polynomials are specializations of the 2 -variable Tutte polynomial $T_{G}(x, y) ;(2.2)$ is a special case of the duality relation satisfied by the Tutte polynomial: $T_{G}(x, y)=T_{G^{*}}(y, x)$. Like the chromatic polynomial, the flow polynomial at positive integers admits a well-known combinatorial interpretation: for $n \in \mathbb{Z}_{+}, F_{G}(n)$ is the number of nowhere-zero flows with values in an abelian group of order $n$, cf. [4].

2.2. The Yamada polynomial $[24] R_{G}(q)$ is an invariant of spatial ribbon graphs $G$, i.e. ribbon graphs embedded in $\mathbb{R}^{3}$. A ribbon graph is an abstract graph $G$ with an embedding into a surface $S$, so that the complement $S \backslash G$ is a disjoint union of 2 -cells. Given such an embedding, a neighborhood of $G$ in $S$ is a compact surface with boundary, which may be thought of as a choice of a 2 -dimensional thickening of $G$. Such a thickening of vertices and edges may be also encoded using cyclic ordering of half-edges incident to each vertex.

The Yamada polynomial is defined using the contraction-deletion rule:

$$
R_{G}(Q)=R_{G / e}(Q)+R_{G \backslash e}(Q),
$$

and a version of the $S O(3)$ Kauffman skein relations (cf. [7, Section 5]) applied to a planar projection of $G$ :

$$
\begin{gathered}
R_{G \amalg \bigcirc}=\left(q+1+q^{-1}\right) R_{G}, \\
R_{\bigotimes}=q R_{\emptyset}+R_{\bigotimes}+q^{-1} R_{\Xi} \\
R_{\bigotimes}=q^{-1} R_{\emptyset}+R_{\bigotimes}+q R_{\Xi}
\end{gathered}
$$

As usual in skein-theoretic definitions, the graphs in each of these equations differ as shown, and are identical outside of the disk. If $G$ has a bridge, $R_{G}$ is set to be zero. The Yamada polynomial is multiplicative under taking disjoint union, and $R_{G \vee H}=-R_{G} R_{H} . \quad R_{G}$ is an invariant of the isotopy class of an embedding of the ribbon graph $G$ into $\mathbb{R}^{3}$. (In terminology of [24, Theorem 2], $R_{G}$ is a regular deformation invariant of a planar diagram of $G$; in [11] this equivalence relation is called rigid vertex isotopy.)

Using just equations (2.4) - (2.6), one gets a (renormalized version of) the $\mathrm{SO}(3)$ Kauffman polynomial of 4-regular ribbon graphs in $\mathbb{R}^{3}$ (cf. [11], [7]). Therefore the Yamada polynomial may be thought of as the SO(3) Kauffman polynomial, extended 
to spatial ribbon graphs of arbitrary vertex degree using the contraction-deletion rule $(2.3)$.

If $G$ is planar, then there are no crossings to resolve, and the Yamada polynomial is determined by the contraction-deletion rule and its loop value (2.4); in this case $F_{G}(Q)=(-1)^{V-E} R_{G}(q)$, where $Q=q+2+q^{-1}$. In general, the Yamada polynomial carries a lot of information about the embedding of $G$ into $\mathbb{R}^{3}$. For example, tying a knot into an edge of $G$ results (up to a normalization) in multiplication of $R_{G}$ by the $\mathrm{SO}(3)$ invariant of the knot. Therefore, in general the Yamada polynomial of a ribbon graph is quite different from the flow polynomial of the underlying abstract graph.

To describe the TQFT context for the results of this paper, next we give a brief summary of the relevant material on the Temperley-Lieb algebra, the chromatic algebra and their structure at roots of unity.

2.3. The Temperley-Lieb algebra. The Temperley-Lieb algebra, $\mathrm{TL}_{n}$, is an algebra over $\mathbb{C}[d]$ consisting of linear combinations of 1-dimensional submanifolds, considered up to isotopy rel boundary, in a rectangle. Each submanifold meets both the top and the bottom of the rectangle in $n$ points. Deleting a simple closed curve has the effect of multiplying the element by $d$. Often $d$ will be specialized to a complex number, and in this case the algebra will be denoted $\mathrm{TL}_{n}^{d}$. The multiplication is given by vertical stacking of rectangles. The standard generators of $\mathrm{TL}_{4}$ are shown in figure 1.

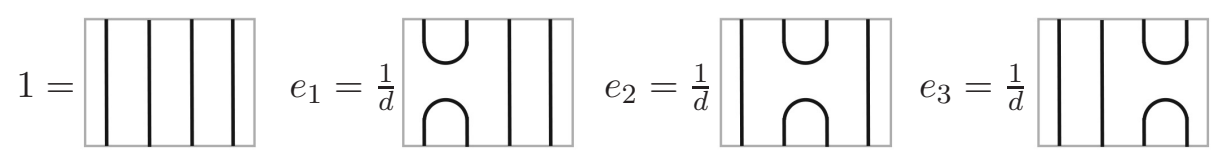

Figure 1. Generators of $\mathrm{TL}_{4}$

The trace $\operatorname{tr}_{d}: \mathrm{TL}_{n}^{d} \longrightarrow \mathbb{C}$ is defined on rectangular pictures by connecting the top and bottom endpoints by disjoint arcs in the complement of the rectangle in the plane, and then evaluating $d^{\# \text { circles }}$. The Hermitian product is defined by $\langle a, b\rangle=\operatorname{tr}(a \bar{b})$, where the involution - reflects pictures in a horizontal line and replaces coefficients with their complex conjugates.

For special values of the parameter $d, d=2 \cos \left(\frac{\pi k}{n+1}\right)$, where $k, n+1$ are coprime, $\mathrm{TL}_{n}^{d}$ contains a non-trivial ideal, the trace radical consisting of the elements $a$ such that $\operatorname{tr}(a b)=0$ for all $b \in \mathrm{TL}_{n}^{d}$. This ideal is generated by the Jones-Wenzl projector $P^{(n)}[23,10]$. At primitive roots of unity $(k=1)$ the Hermitian product descends to a positive definite inner product on the quotient of $\mathrm{TL}_{n}^{d}$ by the trace radical. 
2.4. The chromatic algebra $\mathcal{C}_{\mathbf{n}}$. The Temperley-Lieb algebra, discussed above, underlies the construction of SU(2) TQFT. Next we briefly summarize the definition and properties of the chromatic algebra, $\mathcal{C}_{n}$, corresponding to SO(3) TQFT; we refer the reader to [6, Section 2] for more details. (A similar notion, in a different context, was considered in [12, Section 2].)

$\mathcal{C}_{n}$ is an algebra over $\mathbb{C}[Q]$, whose elements are formal linear combinations of planar cubic graphs (considered up to isotopy rel boundary) in a rectangle, modulo the local relations shown in figure 2. The first relation is the analogue of the contractiondeletion relation for cubic graphs.

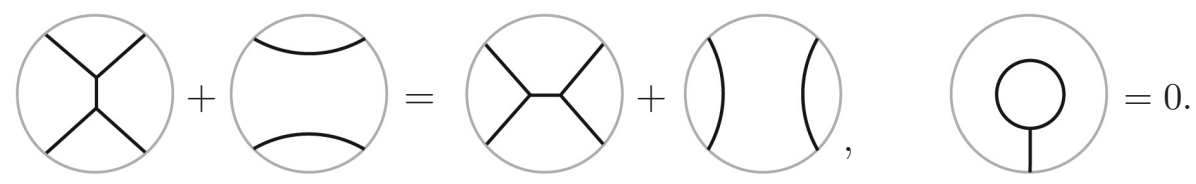

FiguRE 2. Relations defining the chromatic algebra.

The intersection of a graph with the boundary of the rectangle consists of $2 n$ points: $n$ points both at the top and the bottom, figure 3 . It is convenient to allow 2 -valent vertices as well, and the value of a simple closed curve is set to be $Q-1$. When $Q$ is specified to a complex number, the algebra is denoted $\mathcal{C}_{n}^{Q}$.
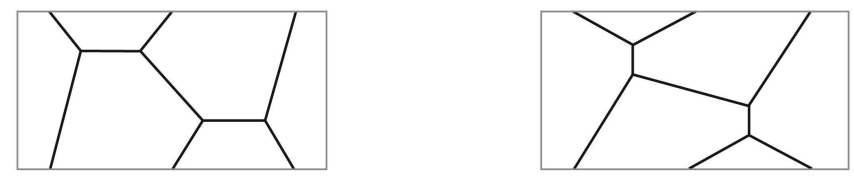

Figure 3. Examples of graphs in $\mathcal{C}_{3}$.

The trace $\operatorname{tr}: \mathcal{C}_{n}^{Q} \longrightarrow \mathbb{C}$ is defined by connecting the endpoints of $G$ by disjoint arcs in the complement of the rectangle in the plane and evaluating the flow polynomial of the resulting graph at $Q$. (Or equivalently, the trace equals $Q^{-1}$ times the chromatic polynomial of the dual graph.) The trace is well-defined since the local relations in figure 2 are precisely the relations defining the flow polynomial of a planar cubic graph. The multiplication and the Hermitian product on $\mathcal{C}_{n}^{Q}$ are defined analogously to those in the Temperley-Lieb algebra.

There are two variations of the definition of the chromatic algebra, which are going to be useful. First, rather than using just cubic graphs modulo relations in figure $2, \mathcal{C}_{n}$ may also be defined using graphs with arbitrary vertex degrees, modulo the contraction-deletion relation, see [6, Section 5].

Second, instead of using planar graphs, one may consider ribbon graphs in the cylinder $D^{2} \times[0,1]$, with $n$ endpoints both at $D^{2} \times 0$ and at $D^{2} \times 1$, modulo the defining 
relations of the Yamada polynomial, (2.3) - (2.6). This is closely related to the definition of the SO(3) BMW algebra (cf. [7, Section 5]). Using the relations

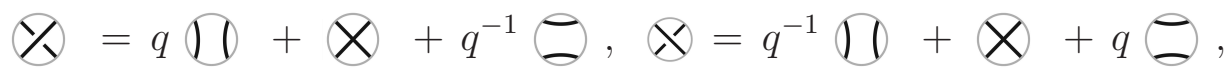

all crossings of a ribbon graph in the cylinder may be resolved to give an element of $\mathcal{C}_{n}$.

2.5. The map $\mathcal{C}_{\mathbf{n}} \longrightarrow \mathrm{TL}_{\mathbf{2 n}}$. Consider the homomorphism $\Phi: \mathcal{C}_{n}^{Q} \longrightarrow \mathrm{TL}_{2 n}^{d}$, where $Q=d^{2}$, replacing each edge of a graph with the second Jones-Wenzl projector, and resolving each vertex as shown in figure 4. Moreover, for a trivalent graph $G$ there is an overall factor $d^{V / 2}$, where $V$ is the number of vertices of $G$.

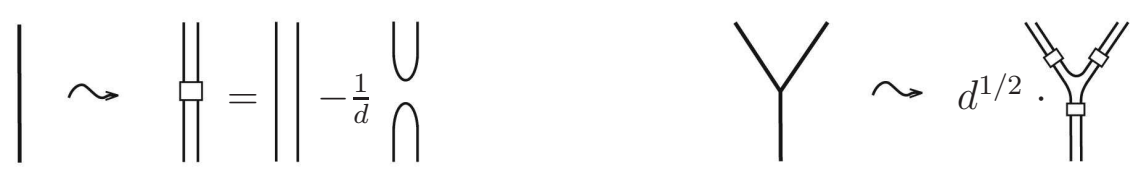

Figure 4. The homomorphism $\Phi: \mathcal{C}_{n}^{Q} \longrightarrow \mathrm{TL}_{2 n}^{d}, Q=d^{2}$

$\Phi$ induces a well-defined homomorphism of algebras $\mathcal{C}_{n}^{Q} \longrightarrow \mathrm{TL}_{2 n}^{d}$, where $Q=d^{2}$, and moreover it is trace-preserving: the diagram

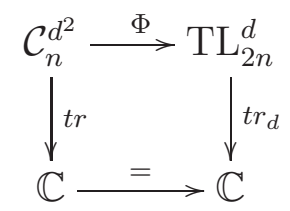

commutes [6, Lemmas 2.4, 2.5]. It follows that the pullback under $\Phi$ of the trace radical in $\mathrm{TL}^{d}$ is in the trace radical of $\mathcal{C}^{d^{2}}$. As mentioned in section 2.3, the trace radical in $\mathrm{TL}^{d}$ is non-trivial precisely for the values $d=2 \cos \left(\frac{\pi k}{n+1}\right)$. The elements of the trace radical in the chromatic algebra $\mathcal{C}^{d^{2}}$ are local relations (which hold in addition to the contraction-deletion rule) on graphs which preserve the flow polynomial, or equivalently the chromatic polynomial of the dual graph. When $d=\phi$, the trace radical of the Temperley-Lieb algebra is generated by the Jones-Wenzl projector $P^{(4)}$. In particular, the linear Tutte relation in $\mathcal{C}_{4}^{Q}$, where $Q=\phi+1=\phi^{2}$,

$$
\text { ( }=\phi^{-1} \cap-\phi^{-2}
$$

may be seen as a consequence of the structure of $\mathrm{TL}^{\phi}$ since it is mapped by $\Phi$ to $P^{(4)}[6$, Section 2, p. 721]. Similarly, the linear relation

$$
\text { ( }=-\phi \sqrt{0}-\phi^{2} \text {, }
$$

holds in $\mathcal{C}_{4}^{Q}$, at the Galois conjugate value $Q=(3-\sqrt{5}) / 2$. 
2.6. The flow category. To study the flow polynomial of non-planar graphs, one may use abstract (not necessarily planar) graphs to define an algebra along the lines of the Temperley-Lieb and chromatic algebras [12, 22]. Unlike the chromatic algebra case, here one does not have a map to the Temperley-Lieb algebra, and the values $Q=d^{2}, d=2 \cos \left(\frac{\pi}{n+1}\right)$ do not play a special role since these are artifacts of planarity. We will not use the algebra structure; the notion of a category is more suitable for our applications. The construction of the flow category is summarized below, following [1, Section 3.1].

The objects of the flow category are finite ordered sets $\bar{n}=\{1, \ldots, n\}$. Consider $\mathcal{G}_{m, n}=\{$ finite graphs with $m+n$ marked univalent vertices $\}$, where the marked vertices are divided into two ordered subsets of $m$, respectively $n$ vertices. The edges incident to the marked vertices are called boundary edges and the rest are internal edges.

The space of morphisms $\mathcal{F}_{m, n}^{Q}$ in the flow category between $\bar{m}, \bar{n}$ consists of formal $\mathbb{C}$ linear combinations of graphs $\mathcal{G}_{m, n}$, modulo the contraction-deletion relation which applies to internal edges, figure 5. Graphs whose equivalence classes are elements of $\mathcal{F}_{m, n}^{Q}$ may be represented geometrically as in figure 5 . (It is important to note that unlike in sections 2.2, 2.4, over/under-crossings do not carry any information here since the figure represents the abstract graph structure and not a specific planar projection.) In addition, the loop value is set to be $Q-1$, and graphs with a univalent vertex (other than the specified marked vertices) are set to be zero.

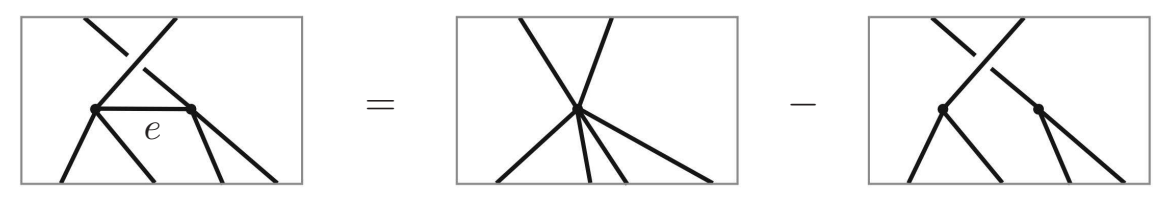

Figure 5. The contraction-deletion rule in $\mathcal{F}_{4,2}$.

A graph without marked vertices, and therefore no boundary edges, considered in $\mathcal{F}_{0,0}^{Q} \cong \mathbb{C}$, evaluates to its flow polynomial at $Q$. The pairing $\mathcal{F}_{k, m}^{Q} \times \mathcal{F}_{m, n}^{Q} \longrightarrow \mathcal{F}_{k, n}^{Q}$ is obtained by gluing along $m$ boundary edges. For example, this pairing applied to two graphs $A \in \mathcal{G}_{0, m}, B \in \mathcal{G}_{m, 0}$ gives $\langle A, B\rangle=$ the value of the flow polynomial $F_{A \cup B}(Q)$.

Given any graph representing an element of $\mathcal{F}_{m, n}^{Q}$, the contraction-deletion rule may be used to eliminate all internal edges. For example, the four graphs in figure 6 form a basis of $\mathcal{F}_{0,4}^{Q}$. Three of these graphs, viewed relative to a fixed embedding of the marked vertices in the boundary, are planar, and one, denoted $e_{4}$, is non-planar. 

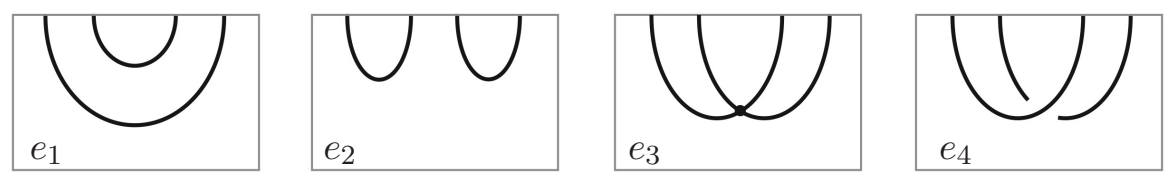

Figure 6. A basis of $\mathcal{F}_{0,4}^{Q}$.

It is convenient to introduce the notation $\mathcal{C}_{m, n}^{Q}$ for the planar analogue of $\mathcal{F}_{m, n}^{Q}$. That is, $\mathcal{C}_{m, n}^{Q}$ consists of formal $\mathbb{C}$-linear combinations of planar graphs with two ordered subsets of $m$, respectively $n$ marked vertices, modulo the contraction-deletion relation which applies to internal edges.

2.7. Conventions and notation. Unless stated otherwise, in the following sections the flow polynomial of planar graphs and the Yamada polynomial of spatial ribbon graphs will be considered in the context of the chromatic algebra. On the other hand, the flow polynomial of abstract (non-planar) graphs does not fit in this context, and it will be studied in the setting of the flow category.

It is convenient to introduce a short-hand notation for the evaluation of graph polynomials. For example, when working with the Yamada polynomial of a graph $G$ with a specified crossing $\mathbb{Q}$, the notation $\mathbb{C}_{x}$ will stand for the evaluation of $R_{G}(x)$. Similarly, given two graphs $G_{1}, G_{2}$ in a disk with the same number of marked points on the boundary, the notation $\left\langle G_{1}, G_{2}\right\rangle_{Q}$ will stand for the relevant pairing. For example, in the context of the flow category, it will mean the value of the flow polynomial $F_{G_{1} \cup G_{2}}$ at $Q$, where the union of $G_{1}, G_{2}$ is taken along the marked points on the boundary.

\section{The GOLDEN IDENTITY FOR THE YAMADA POLYNOMIAL}

The purpose of this section is to prove the extension (1.2) of the Tutte golden identity (1.1) to the Yamada polynomial of cubic ribbon graphs in $\mathbb{R}^{3}$. It is convenient to allow vertices of degree 2 in the statement of the theorem:

Theorem 3.1. Let $G$ be a ribbon graph in $\mathbb{R}^{3}$, with vertices of degrees 2 and 3 . Then

$$
R_{G}\left(e^{\pi i / 5}\right)=(-1)^{V-E} \phi^{E^{\prime}} R_{G}\left(e^{-2 \pi i / 5}\right)^{2},
$$

where $E^{\prime}=V_{3}-\chi(G), V_{3}$ is the number of trivalent vertices of $G, \chi(G)$ is the Euler characteristic, and $\phi=(1+\sqrt{5}) / 2$.

If there are no vertices of degree 2 , then $E^{\prime}=E$, and (3.1) is the same identity as (1.2). Moreover, since both $\chi(G)$ and $V_{3}(G)$ are topological invariants of $G$, introducing new 2 -valent vertices (i.e. subdividing edges of $G$ ) does not affect (3.1). 
In the special case where $G$ has no trivalent vertices, (3.1) gives an identity for the SO (3) Kauffman polynomial of framed links in $\mathbb{R}^{3}$, where the factor $(-1)^{V-E} \phi^{E^{\prime}}$ equals 1. Then the left-hand side of (3.1) may be interpreted as the 2-colored Jones polynomial of the link, and the right-hand side equals the square of the Jones polynomial. Therefore for links, (3.1) matches the identity stated in [13, Corollary 4.16].

Proof of theorem 3.1. Recall from section 2.2 that if $G$ is planar, one has $F_{G}(Q)=$ $(-1)^{V-E} R_{G}(q)$, where $Q=q+2+q^{-1}$. In particular, for planar graphs $G$,

$$
R_{G}\left(e^{2 \pi i / 10}\right)=F_{G}(\phi+2), R_{G}\left(e^{-2 \pi i / 5}\right)=F_{G}(\phi+1) .
$$

Therefore in this case, (3.1) is reduced to the Tutte golden identity (1.1). ${ }^{1}$ In the general case of a ribbon graph $G$ in the statement of the theorem, the proof is by induction on the number $c$ of crossings in a planar diagram of $G$. The base case corresponds to planar graphs, discussed above.

As in section 2.7, the notation $\bigcirc_{q}$ in the proof below will denote the evaluation of the Yamada polynomial at $q$.

By induction assume that graphs with fewer than $c$ crossings satisfy (3.1). Consider a graph with $c$ crossings. For brevity of notation denote $x:=e^{2 \pi i / 10}, y:=e^{-2 \pi i / 5}$. Combining the skein relation (2.5) with the contraction-deletion rule (2.3), one has

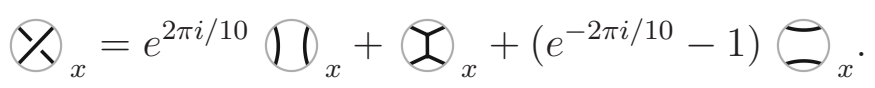

Since the three graphs on the right have $c-1$ crossings, (3.1) holds for them by the inductive assumption, thus

$$
\bigotimes_{x}=(-1)^{V-E} \phi^{E^{\prime}}\left(e^{2 \pi i / 10} \bigoplus_{y}^{2}-\phi^{3} \boldsymbol{\Upsilon}_{y}^{2}+\left(e^{-2 \pi i / 10}-1\right) \boldsymbol{\sigma}_{y}^{2}\right) \text {. }
$$

Because of the normalization sign $(-1)^{V-E}$ in the formula relating the flow and Yamada polynomials of planar graphs, the linear relation (2.8) at $q=e^{-2 \pi i / 5}$ (corresponding to $Q=\phi+1$ ) for the Yamada polynomial reads

$$
\Upsilon_{y}=-\phi^{-1} \bigcap_{y}+\phi^{-2} \sigma_{y}
$$

Applying this relation to $\mathbf{Y}_{y}$ in $(3.3)$ gives

$$
\bigotimes_{x}=(-1)^{V-E} \phi^{E^{\prime}}\left(\left(e^{2 \pi i / 10}-\phi\right) \bigoplus_{y}^{2}+2 \bigcap_{y} \sigma_{y}+\left(e^{-2 \pi i / 10}-\phi\right) \boldsymbol{\sigma}_{y}^{2}\right)
$$

\footnotetext{
${ }^{1}$ Its proof is given in section 7.
} 
To prove the inductive step, one needs to show that $(3.5)$ equals $(-1)^{V-E} \phi^{E^{\prime}} \bigotimes_{y}^{2}$. Using the skein relation (2.5) and the contraction-deletion rule,

$$
(-1)^{V-E} \phi^{E^{\prime}} \bigotimes_{y}^{2}=(-1)^{V-E} \phi^{E^{\prime}}\left(e^{-2 \pi i / 5} \bigoplus_{y}+\boldsymbol{\Upsilon}_{y}+\left(e^{2 \pi i / 5}-1\right) \boldsymbol{\sigma}_{y}\right)^{2} .
$$

To complete the proof, one replaces $\int_{y}$ with (3.4), and checks that the resulting expression matches (3.5).

\section{Structure of the flow polynomial (MOD 5)}

In this section we establish the $(\bmod 5)$ version of the golden identity, and prove a theorem stated in the introduction:

Theorem 1.2. Let $G$ be a cubic graph with $V$ vertices. Then the value of the flow polynomial of $G$ at zero satisfies:

$$
\begin{gathered}
F_{G}(0) \equiv 0,1,4(\bmod 5) \text { if } V / 2 \text { is even, } \\
F_{G}(0) \equiv 0,2,3(\bmod 5) \text { if } V / 2 \text { is odd. }
\end{gathered}
$$

Moreover, suppose $G$ is a snark, that is a bridgeless cubic graph with chromatic index 4. Then $F_{G}(0) \equiv 0(\bmod 5)$, and therefore $F_{G}(0)$ is divisible by 120 .

Remark. [4, Theorem 1.2] interpreted $\left|F_{G}(0)\right|$ as the number of Eulerian equivalence classes of totally cyclic orientations.

Proof of theorem 1.2. We start by stating the $(\bmod 5)$ version of the golden identity:

Lemma 4.1. For planar cubic graphs $G$,

$$
F_{G}(0) \equiv 3^{E} F_{G}(4)^{2}(\bmod 5),
$$

where $E$ is the number of edges of $G$. More generally, let $G$ be a ribbon cubic graph in $\mathbb{R}^{3}$. Then

$$
R_{G}(-1) \equiv(-1)^{V-E} 3^{E} R_{G}(1)^{2}(\bmod 5)
$$

Proof of (4.3). The flow polynomial $F_{G}(Q)$ for $Q=(3-\sqrt{5}) / 2,(5-\sqrt{5}) / 2$ takes values in the ring $R=\mathbb{Z}\left[\frac{1+\sqrt{5}}{2}\right]$. Consider the ideal $I$ generated by $\sqrt{5}$. Since $\phi \sqrt{5}=(5+\sqrt{5}) / 2 \in I$, it follows that $1+\phi \equiv 4(\bmod \sqrt{5})$, and $\phi \equiv 3(\bmod \sqrt{5})$. Plugging this into the golden identity $(1.1)$, one gets $F_{G}(0) \equiv 3^{E} F_{G}(4)^{2}(\bmod \sqrt{5})$. Since $F_{G}(0)$ and $3^{E} F_{G}(4)^{2}$ are integers, the equivalence holds $(\bmod 5)$.

Proof of (4.4). The values of the Yamada polynomial $R_{G}\left(e^{\pi i / 5}\right), R_{G}\left(e^{-2 \pi i / 5}\right)$ are elements of $\mathbb{Z}\left[\zeta_{5}\right]$, where $\zeta_{5}=e^{\pi i / 5}$. Consider the ideal generated by $e^{\pi i / 5}+1$. Modulo this ideal, $e^{\pi i / 5}$ and $e^{-\pi i / 5}$ are equivalent to $-1, e^{2 \pi i / 5}$ and $e^{-2 \pi i / 5}$ are equivalent to 1 , and $\phi=e^{\pi i / 5}+e^{-\pi i / 5} \equiv 3$. 
Remark. Another proof of lemma 4.1 may be given by following (mod 5) the steps of the proofs of the golden identities (1.1), (3.1). For example, the (mod 5 ) version at $Q=4$ of the Tutte linear relation (2.8) for the flow polynomial of planar graphs, $\Upsilon_{\phi+1}=\phi^{-1} \bigoplus_{\phi+1}-\phi^{-2} \varrho_{\phi+1}$, reads $\boldsymbol{\Upsilon}_{4} \equiv 2 \bigoplus_{4}+\boldsymbol{\sigma}_{4}(\bmod 5)$.

Returning to the proof of theorem 1.2, recall that for planar graphs $G, F_{G}(Q)=$ $(-1)^{V-E} R_{G}(q)$, where $Q=q+2+q^{-1}$. In particular, for planar $G, R_{G}(-1)=$ $(-1)^{V-E} F_{G}(0)$. The following identity holds for the flow polynomial of all (planar and non-planar) graphs:

$$
\bigotimes_{0}=-D_{0}-\bigotimes_{0}-\ddot{\sigma}_{0}
$$

This identity is checked by pairing both sides of (4.5) with the four basis elements of the flow category $\mathcal{F}_{0,4}^{Q}$ in figure 6 . This relation coincides with the skein relation (2.5) for the Yamada polynomial, normalized by the factor $(-1)^{V-E}$, at $q=-1$. Thus $R_{G}(-1)=(-1)^{V-E} F_{G}(0)$ for all graphs $G$. Now it follows from (4.4) that for any abstract (planar or non-planar) cubic graph $G$,

$$
F_{G}(0) \equiv 3^{E} R_{G}(1)^{2}(\bmod 5) \text {. }
$$

Remark. Up to a sign, the value $R_{G}(1)$ of the Yamada polynomial of ribbon cubic graphs equals the Penrose number of $G$, introduced and studied in [14], [9, Section 2.3]. Indeed, the skein relation [9, Proposition 2], satisfied by the Penrose number, coincides with the version of the skein relation $(2.5)$ for $(-1)^{V-E} R_{G}(1)$ :

$$
\Phi_{1}=Q_{1}-Q_{1}
$$

It is an invariant of ribbon graphs where the ribbon structure affects only its sign, so $\left|R_{G}(1)\right|$ is an invariant of abstract cubic graphs.

The congruence (4.6), the relation $3 V=2 E$ for cubic graphs, and the fact that perfect squares are congruent to 0,1 , or $4(\bmod 5)$ conclude the proof of $(4.1),(4.2)$.

To prove the last statement of the theorem, consider a planar diagram of a ribbon cubic graph $G$. In other words, the graph is immersed in the plane, with some edge crossings. The value $R_{G}(1)$ is independent of which strand is over/under in each crossing. Using the recursion relation (4.7) at $q=1$, one checks that $(-1)^{V-E} R_{G}(1)$ equals the signed count of 3 -edge colorings of $G$, where the sign gets a $(-1)$ factor for each pair of edges that cross with a different color. This is invariant of regular homotopy of the planar diagram, and it satisfies the skein relation: each 3-coloring of the graph on the left in (4.7) corresponds (with the same sign) to a 3 -coloring of precisely one term on the right.

This implies that the Yamada polynomial of snarks at $q=1$ is zero. (More generally, there is a well-known correspondence between 3 -edge-colorings and 4-flows, cf. Proposition 6.4 .5 (ii) in [5], so for any cubic graph $\left|R_{G}(1)\right| \leq F_{G}(4)$, with equality for 
planar graphs.) Now the congruence $F_{G}(0) \equiv 0(\bmod 5)$ follows from $(4.6)$. Finally, since the flow polynomial $F_{G}(Q)$ of a snark is divisible by $\prod_{k=1}^{4}(Q-k), F_{G}(0)$ is also divisible by 4 !.

\section{Golden INEQUALity FOR NON-PLANAR CUBiC GRAPHS}

Unlike the golden identity for the Yamada polynomial, Theorem 3.1, which holds for any spatial cubic graph $G$, in [1] we stated a conjecture that the golden identity for the flow polynomial characterizes planarity:

Conjecture 5.1. For any cubic bridgeless graph $G$,

$$
(-\phi)^{E} F_{G}((5-\sqrt{5}) / 2) \geq F_{G}((3-\sqrt{5}) / 2)^{2},
$$

Moreover, $G$ is planar if and only if (5.1) is an equality.

In this section we develop methods to prove this conjecture for a certain family of non-planar cubic graphs. We will call a graph $G$ near-planar if it admits a planar projection with a single crossing, for example $K_{3,3}$ in figure 7 . We will view such graphs as $G=\bigotimes \cup \bar{G}$ where $\bar{G}$ is a planar graph in a disk (the complement in $S^{2}$ of the shaded disk in figure 7) with 4 endpoints on the boundary circle.

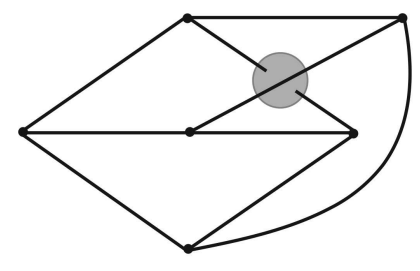

FiguRE 7.

Considered as an element of the chromatic algebra $C_{2}^{Q}, \bar{G}$ may be expressed as a linear combination of the three basis elements without internal edges. The coefficients depend on $Q$; denote by $\alpha, \beta, \gamma$ their values at $Q=(3-\sqrt{5}) / 2$, figure 8 . In the following lemma the graph $\bar{G}$ outside a disk will be fixed throughout the proof, and (as in section 2.7) the notation $\bigcirc_{Q}$ will stand for the evaluation of the flow polynomial at $Q$.

Lemma 5.2 (Golden identity for the flow polynomial of near-planar graphs). Let $G=\mathscr{G} \cup \bar{G}$ be a near-planar graph. Then the following identity holds for the evaluations of the flow polynomial of $G$ and of the two planar graphs obtained from $G$ by resolving the crossing:

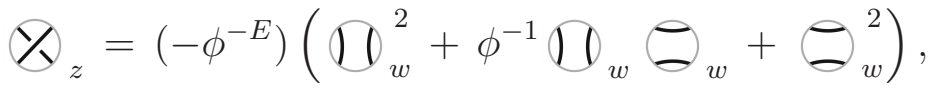

where $z=(5-\sqrt{5}) / 2, w=(3-\sqrt{5}) / 2$. 


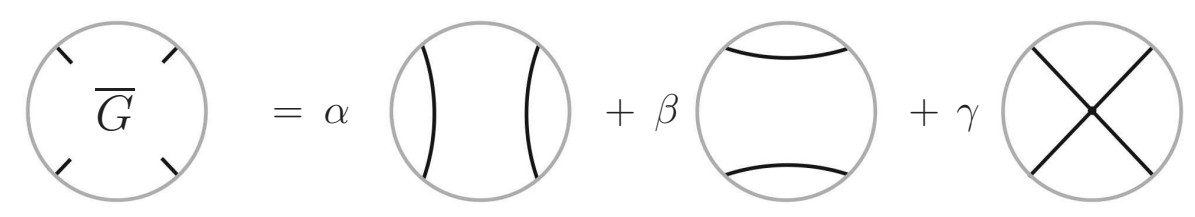

Figure 8. A planar cubic graph in $C_{2}^{Q}, Q=(3-\sqrt{5}) / 2$, expressed as a linear combination of basis vectors.

We remark that this lemma is motivated by D.W. Hall's version of the golden identity for constrained chromials [8]. The proof of lemma 5.2 relies on the following statement.

Proposition 5.3. Fix $Q \neq(3 \pm \sqrt{5}) / 2$, and let $G=\bigotimes \cup \bar{G}$ be a near-planar graph. Then

$F_{G}(Q)=\bigotimes_{Q}=-\frac{1}{Q^{2}-3 Q+1} \bigoplus_{Q}+\frac{Q-1}{Q^{2}-3 Q+1} \bigotimes_{Q}-\frac{1}{Q^{2}-3 Q+1} \sigma_{Q}$

The proof of proposition 5.3 amounts to checking that both sides have identical evaluations when paired up with the three basis elements $\square, \varnothing$ and $\bigotimes$ of the chromatic algebra $\mathcal{C}_{2}^{Q}$. (As discussed in section 2.5, the bilinear pairing $\langle.,\rangle_{Q}$ on $C_{2}^{Q}$ is non-degenerate precisely for $Q \neq(3 \pm \sqrt{5}) / 2$.)

Proof of lemma 5.2. Setting $Q$ to equal $z=(5-\sqrt{5}) / 2$ in proposition 5.3 and using the contraction-deletion rule, one has the following equality:

$$
\bigotimes_{z}=\frac{\phi}{2} \bigoplus_{z}-\frac{\phi^{-1}}{2} \boldsymbol{\zeta}_{z}+\frac{1}{2} \sigma_{z}
$$

Equation (5.2) is obtained by applying the golden identity (1.1) to the three planar cubic graphs on the right in (5.3).

It follows from lemma 5.2 that conjecture 5.1 for near-planar graphs is equivalent to the inequality

$$
\bigotimes_{w}^{2} \leq \bigoplus_{w}^{2}+\sigma_{w}^{2}+\phi^{-1} \bigcap_{w} \sigma_{w}
$$

Remark 5.1. It is interesting to note that the inequality (5.4) can be restated in terms of the Yamada polynomial:

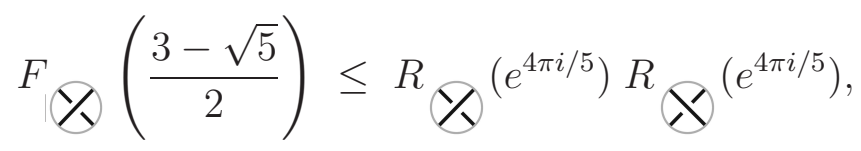

where the left-hand side is the evaluation of the flow polynomial of the abstract nearplanar graph, and the right-hand side is the product of the Yamada polynomials of two spatial ribbon graphs corresponding to the two possible crossings. The proof 
of the equivalence of (5.4), (5.5) consists of applying the Yamada polynomial skein relations to the crossings, and simplifying using the linear relation (2.9).

We are now in a position to give a reformulation of conjecture 5.1 for near-planar graphs.

Lemma 5.4. Let $\bar{G}$ be a cubic graph in a disk, with 4 marked points on the boundary. Considered in the chromatic algebra $C_{2}^{Q}, Q=(3-\sqrt{5}) / 2, \bar{G}=\alpha \emptyset+\beta-$ $\gamma \bigotimes$. Conjecture 5.1 for near-planar graphs is equivalent to the inequality

$$
(1+3 \phi) \alpha \beta \leq \gamma(\alpha+\beta+\gamma)
$$

Proof. The term being squared on the right-hand side of (5.1) equals:

$$
\begin{gathered}
F_{G}(w)=\langle\bigotimes, \bar{G}\rangle_{w}=\langle\bigotimes, \alpha \emptyset+\beta \Xi+\gamma \bigotimes\rangle_{w} \\
=-\phi^{-1}(\alpha+\beta)+\phi^{-2} \gamma .
\end{gathered}
$$

Analogous calculations for $\Phi$ and $\sigma$ in place of $\varnothing$ yield

$$
-\phi^{-1} \beta+\phi^{-2}(\alpha+\gamma), \quad-\phi^{-1} \alpha+\phi^{-2}(\beta+\gamma)
$$

respectively. Equation (5.2) expresses the left-hand side in (5.1) in terms of $\square_{w}$ and $\sigma_{w}$. Multiplying out the resulting expressions, (5.1) is seen to be equivalent to $(5.6)$.

Next we establish the inequality (5.6) for an infinite family of near-planar graphs.

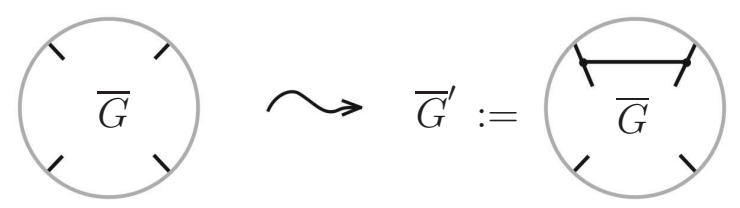

FIGURE 9. A modification of $\bar{G}$ : addition of a peripheral edge (connecting two boundary edges of a cubic graph $\bar{G}$ ).

Lemma 5.5. Conjecture 5.1 holds for the family of near-planar graphs $G=\bigotimes \cup \bar{G}$, where $\bar{G}$ is inductively built by addition of peripheral edges.

Examples of graphs, considered in this lemma, are shown in figure 10. The graph on the left, capped with (1.4) at the Galois conjugate values $\phi+1, \phi+2$ also holds for the same family of graphs. 

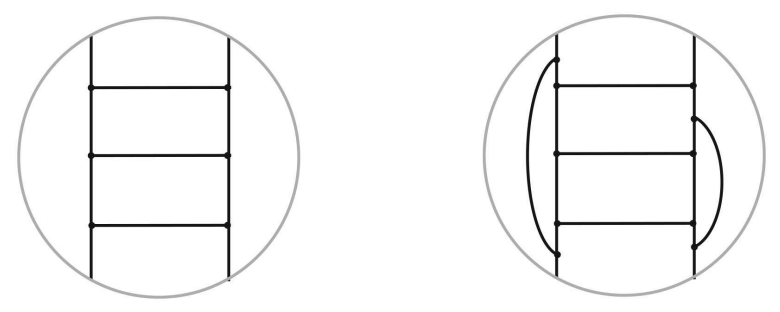

Figure 10. Examples of graphs $\bar{G}$ in lemma 5.5.

Proof of lemma 5.5. It suffices to prove that the inequality (5.6) is preserved under addition of a peripheral edge. We will also show that whenever the coefficients $\alpha, \beta, \gamma$ are non-zero, they satisfy

$$
\operatorname{sign}(\alpha)=\operatorname{sign}(\beta)=-\operatorname{sign}(\gamma)=(-1)^{V / 2},
$$

where $V$ is the number of vertices of $\bar{G}$. The result of adding a peripheral edge to $\bar{G}=\alpha \sqrt[D]{ }+\beta(\bar{\sigma})+\gamma \bigotimes$ is the graph $\bar{G}^{\prime}$ shown in figure 11. (There are 4 possible peripheral edges; by symmetry between $\alpha$ and $\beta$ the proof below applies to each one.)

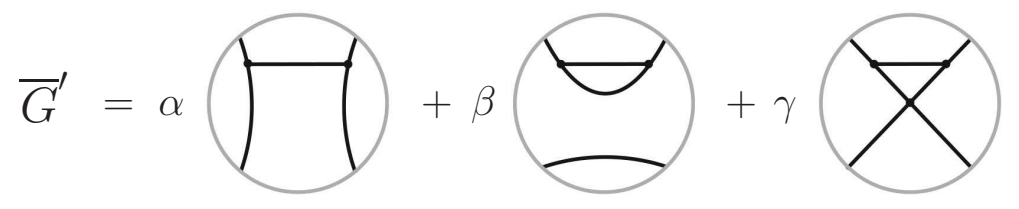

Figure 11. A peripheral edge added to $\bar{G}$.

Applications of the contraction-deletion rule give

$$
\bar{G}^{\prime}=-\alpha \emptyset+(-\phi \beta+\gamma) \boldsymbol{\sigma}+\left(\alpha-\phi^{2} \gamma\right) \bigotimes
$$

Denote the coefficients of $\bar{G}^{\prime}$ by $\alpha^{\prime}, \beta^{\prime}, \gamma^{\prime}$ :

$$
\alpha^{\prime}=-\alpha, \beta^{\prime}=-\phi \beta+\gamma, \gamma^{\prime}=\alpha-\phi^{2} \gamma \text {. }
$$

This gives an inductive proof of the statement (5.7). A direct calculation shows that the desired inequality for the coefficients $\alpha^{\prime}, \beta^{\prime}, \gamma^{\prime}$ of $\bar{G}^{\prime}$,

$$
(1+3 \phi) \alpha^{\prime} \beta^{\prime} \leq \gamma^{\prime}\left(\alpha^{\prime}+\beta^{\prime}+\gamma^{\prime}\right)
$$

is equivalent to

$$
\phi^{2} \alpha \beta \leq \gamma(\alpha+\beta+\gamma)
$$

Since $\operatorname{sign}(\alpha)=\operatorname{sign}(\beta), \phi^{2} \alpha \beta \leq(1+3 \phi) \alpha \beta$.Therefore the assumed inequality (5.6) for $\alpha, \beta, \gamma$ implies the inequality (5.9) for $\alpha^{\prime}, \beta^{\prime}, \gamma^{\prime}$. 
Finally, consider the last statement in conjecture 5.1. Note that the inequality $\phi^{2} \alpha \beta \leq(1+3 \phi) \alpha \beta$ in the previous paragraph is strict precisely when both $\alpha, \beta$ are non-zero. Suppose the inductive construction of the family of graphs in the statement of the lemma starts with $\beta$, and consider the first time a horizontal peripheral edge is added. Before this step, the graph $\bar{G}$ is of the form shown on the left in figure 12: there is a vertical line in the disk, intersecting the graph $\bar{G}$ in a single edge. The vector space of graphs in a disk with three boundary points, modulo the defining local relations of the chromatic algebra, is 1-dimensional. Hence it is clear that $\bar{G}=\lambda \otimes$ in $\mathcal{C}_{2}$, and the coefficient $\beta$ (defined in figure) 8 is zero. Then
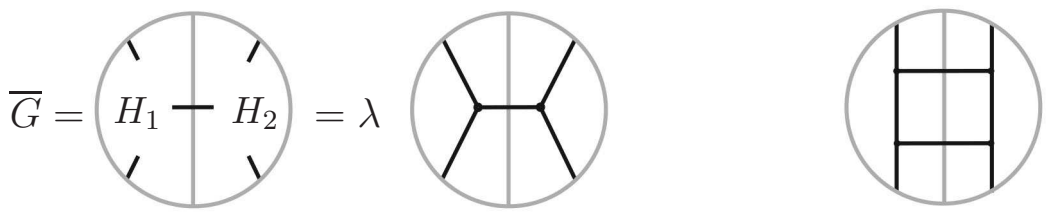

FigURE 12.

adding a horizontal edge gives a scalar multiple of the graph on the right in figure

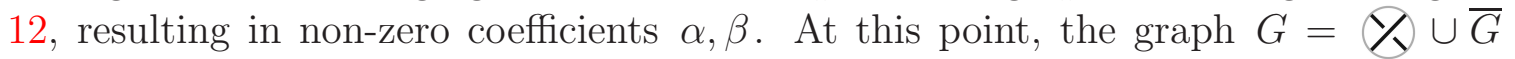
is still planar, since one of the crossing strands may be drawn within the rectangle in the graph on the right in the figure. Since both coefficients $\alpha, \beta$ are non-zero, the addition of any new peripheral edge after this step makes the inequality (5.9) strict. (And of course the graph becomes non-planar.) It follows from (5.8) that all further additions of edges increase $\alpha, \beta$ in absolute value, so the difference of the two sides of the inequality (5.9) continues strictly increasing. Thus the inequality detects planarity in this family of graphs.

\section{Exponential GROWTH OF THE NUMBER OF CHROMATIC POLYNOMials}

In this section we prove theorem 1.3, working dually with the flow polynomial of planar cubic graphs. Consider $\mathcal{C}_{1,3}^{Q}$, the vector space consisting of $\mathbb{C}$-linear combinations of planar cubic graphs in a rectangle with one marked point at the bottom and three marked points at the top of the rectangle, modulo the relations in figure 2. (This notion was also discussed at the end of section 2.6.) The loop value is $Q-1$, and for the remainder of this section we fix $Q=(3-\sqrt{5}) / 2$. As discussed in section 2.5 , at this value of $Q$ there is an additional local relation

$$
\boldsymbol{Y}=-\phi \sqrt{0}-\phi^{2}
$$

$\mathcal{C}_{1,3}^{Q}$ is a module over the chromatic algebra; the action is by vertical concatenation, matching three marked points on the boundary. 


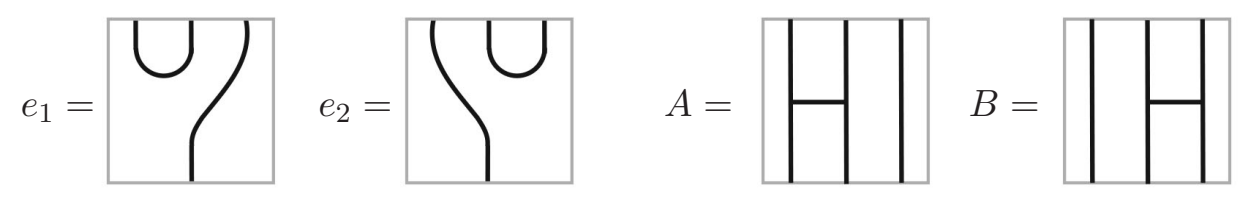

Figure 13. Elements $e_{1}, e_{2} \in \mathcal{C}_{1,3}^{Q}$ and $A, B \in \mathcal{C}_{3}^{Q}$

For a generic $Q$, the vector space $\mathcal{C}_{1,3}^{Q}$ is 3 -dimensional. At the specified value $Q=(3-\sqrt{5}) / 2$, the additional relation (6.1) reduces the dimension to two. Consider its basis $e_{1}, e_{2}$, shown on the left in figure 13 , and let $A, B$ be elements of $\mathcal{C}_{3}$ shown on the right in the same figure. The action of $A, B$ on $e_{1}, e_{2}$ is calculated in figures $14,15$.

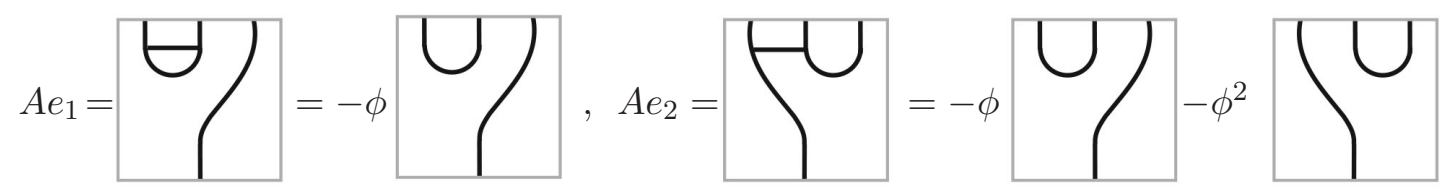

Figure 14. The action of $A$

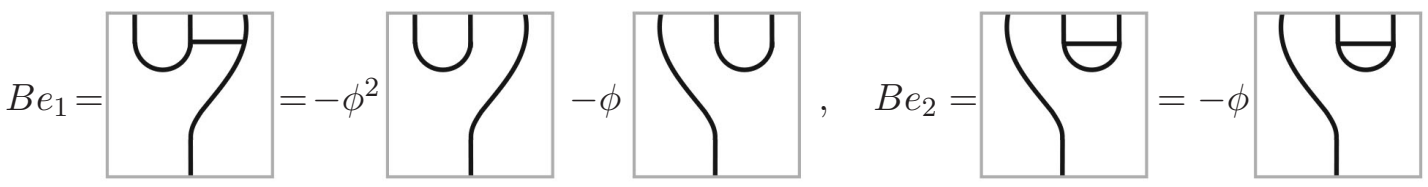

FiguRE 15. The action of $B$

In other words, with respect to the chosen basis, $A$ and $B$ are represented by the matrices

$$
A=-\phi\left[\begin{array}{ll}
1 & 1 \\
0 & \phi
\end{array}\right], B=-\phi\left[\begin{array}{ll}
\phi & 0 \\
1 & 1
\end{array}\right] .
$$

The squares of these matrices are given by

$$
A^{2}=\phi^{2}\left[\begin{array}{ll}
1 & \phi^{2} \\
0 & \phi^{2}
\end{array}\right], B^{2}=\phi^{2}\left[\begin{array}{ll}
\phi^{2} & 0 \\
\phi^{2} & 1
\end{array}\right] .
$$

It follows from the ping pong lemma for semigroups that $A^{2}, B^{2}$ generate a free semigroup in the chromatic algebra $\mathcal{C}_{3}^{Q}$. (Consider vectors $v$ with positive components with respect to the basis $e_{1}, e_{2}$, and let $u=A^{2} v, w=B^{2} v$. Then the components of $u, w$ satisfy $u_{1}>u_{2}, w_{1}<w_{2}$.) Therefore words $\{W\}$ of length $n$ in $A^{2}, B^{2}$ represent $2^{n}$ distinct elements in $\mathcal{C}_{3}^{Q}$. 
Given two elements $a, b \in \mathcal{C}_{1,3}^{Q}$, the product $a \cdot \bar{b}$ (where $\bar{b} \in \mathcal{C}_{3,1}^{Q}$ is obtained from $b$ by reflection in a horizontal line) is an element of $\mathcal{C}_{1}^{Q}$. Here the product is given by vertical concatenation, matching three marked boundary points. $\mathcal{C}_{1}$ is 1 -dimensional, and the trace $\mathcal{C}^{Q} \longrightarrow \mathbb{C}$ is an isomorphism. Denote $\langle a, b\rangle:=\operatorname{tr}(a \cdot \bar{b})$. Note that the Gram matrix $\left\langle e_{i}, e_{j}\right\rangle_{i, j \in\{1,2\}}$ is non-degenerate at $Q=(3-\sqrt{5}) / 2$. Therefore if two words $W_{1}, W_{2}$ in $A^{2}, B^{2}$ are not equal as $2 \times 2$ matrices, then $\left\langle W_{1} e_{i}, e_{j}\right\rangle \neq\left\langle W_{2} e_{i}, e_{j}\right\rangle$ for some $i, j \in\{1,2\}$. Words in $A^{2}, B^{2}$ are represented geometrically by planar cubic graphs $G$ in a rectangle, and (as discussed in section 2.4) $\left\langle W e_{i}, e_{j}\right\rangle$ is the flow polynomial of the graph obtained by gluing $e_{i}$ on the bottom of $G, \bar{e}_{j}$ on top, and then taking the trace, i.e. connecting the endpoints by an arc in the plane, figure 16 .

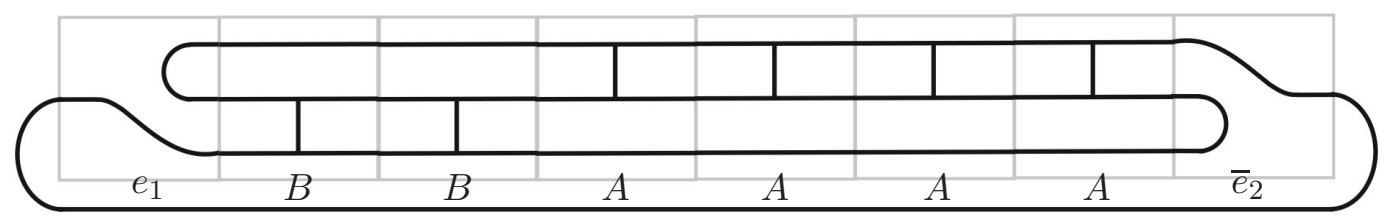

FiguRE 16. The flow polynomial of the pictured graph at $(3-\sqrt{5}) / 2$ equals $\left\langle A^{4} B^{2} e_{1}, e_{2}\right\rangle$. (The multiplication is represented horizontally, rather than vertically, to conserve space.)

Since words of length $n$ in $A^{2}, B^{2}$ give $2^{n}$ distinct matrices, there are at least $2^{n / 4}$ distinct values for one of the matrix entries. This translates into at least $2^{n / 16}$ values $F_{G}((3-\sqrt{5}) / 2)$ of the flow polynomial of planar trivalent graphs $G$ with $n$ vertices.

Remark: After the paper was written, we discovered that one may also prove exponential growth at $Q=4$, observing that $A^{2} e_{1}=4 e_{1}, A^{2} e_{2}=e_{1}+e_{2}, B^{2} e_{1}=$ $e_{1}+e_{2}, B^{2} e_{2}=4 e_{2}$, and completing the argument as above.

\section{Appendix: A proof of the GOLDEN IDENTITy FOR PlANAR CUBIC GRAPHS}

This section gives a variation of the proof [6] of the Tutte golden identity [20] for the flow polynomial of planar cubic graphs:

$$
F_{G}((5-\sqrt{5}) / 2)=(-\phi)^{-E} F_{G}((3-\sqrt{5}) / 2)^{2},
$$

We include a proof since this identity underlies several results in this paper. The version of the proof presented here has more of a computational flavor; it might be useful in numerical investigation of whether there are identities at other parameter values.

Denote $z:=(5-\sqrt{5}) / 2, w:=(3-\sqrt{5}) / 2$. Let $\bar{G}$ be a graph in the disk with 4 marked points on the boundary, and consider its coefficients with respect to the usual 
basis of $C_{2}^{Q}$ (as in figure 8),

$$
\bar{G}=\alpha_{Q} \bigoplus_{Q}+\beta_{Q} \ddot{\sigma}_{Q}+\gamma_{Q} \otimes_{Q}
$$

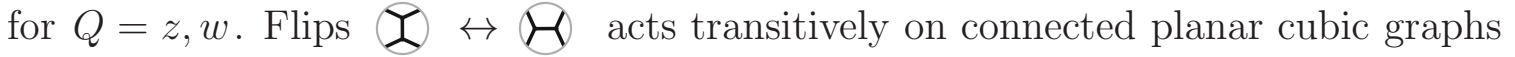
with a fixed number of vertices, and it suffices to prove that the golden identity for (1), and 9 imply the golden identity for $B$.

Consider the evaluations:

$$
\begin{aligned}
& \left\langle(0, \bar{G}\rangle_{Q}=\alpha_{Q}(Q-1)^{2}+\beta_{Q}(Q-1)+\gamma_{Q}(Q-1)^{2},\right. \\
& \langle\Xi, \bar{G}\rangle_{Q}=\alpha_{Q}(Q-1)+\beta_{Q}(Q-1)^{2}+\gamma_{Q}(Q-1)^{2} \text {, } \\
& \langle\mathcal{Y}, \bar{G}\rangle_{Q}=\alpha_{Q}(Q-1)(Q-2)+\gamma_{Q}(Q-1)(Q-2)^{2} \text {, } \\
& \langle\Theta, \bar{G}\rangle_{Q}=\beta_{Q}(Q-1)(Q-2)+\gamma_{Q}(Q-1)(Q-2)^{2} .
\end{aligned}
$$

The golden identity for $G=\bar{G}$, capped off with $\square, G, \mathcal{Q}, \boldsymbol{Q}$, respectively, is equivalent to quadratic equations on the coefficients at $z$ and $w$ :

$$
\begin{gathered}
\alpha_{z} \phi^{-4}+\beta_{z} \phi^{-2}+\gamma_{z} \phi^{-4}-\left(\alpha_{w} \phi^{-2}-\beta_{w} \phi^{-1}+\gamma_{w} \phi^{-2}\right)^{2}=0 \\
\alpha_{z} \phi^{-2}+\beta_{z} \phi^{-4}+\gamma_{z} \phi^{-4}-\left(-\alpha_{w} \phi^{-1}+\beta_{w} \phi^{-2}+\gamma_{w} \phi^{-2}\right)^{2}=0 \\
-\alpha_{z} \phi^{-3}+\gamma_{z} \phi^{-4}-\left(\alpha_{w}-\gamma_{w} \phi\right)^{2}=0 \\
-\beta_{z} \phi^{-3}+\gamma_{z} \phi^{-4}-\left(\beta_{w}-\gamma_{w} \phi\right)^{2}=0
\end{gathered}
$$

The left-hand sides of these four equations satisfy the relation $\left(\phi^{-2}-\phi^{-4}\right)(A-B)=$ $C-D$, so the validity of any three of them implies the fourth. The proof of (7.1) is completed by comparing the loop values: $\bigcirc_{z}=\bigcirc_{w}^{2}$.

Acknowledgements. We would like to thank Gordon Royle for many discussions, and also for sharing with us numerical data on graph polynomials. We also thank Kyle Miller for helpful comments.

V. Krushkal was supported in part by NSF grant DMS-1612159. Ian Agol was supported by NSF grant DMS-1406301, a Simons Investigator Award, and the Institute for Advanced Study. 


\section{REFERENCES}

[1] I. Agol and V. Krushkal, Tutte relations, TQFT, and planarity of cubic graphs, Illinois J. Math. 60 (2016), no. 1, 273-288.

[2] S. Beraha, Infinite non-trivial families of maps and chromials, Thesis, Johns Hopkins University, 1975.

[3] G.D. Birkhoff and D.C. Lewis, Chromatic polynomials, Trans. Amer. Math. Soc. 60 (1946), $355-451$.

[4] B. Chen and R. Stanley, Orientations, lattice polytopes, and group arrangements II: Modular and integral flow polynomials of graphs, Graphs Combin. 28 (2012), no. 6, 751-779.

[5] R. Diestel, Graph theory. Graduate Texts in Mathematics, 173. Springer-Verlag, Berlin, 2005.

[6] P. Fendley and V. Krushkal, Tutte chromatic identities from the Temperley-Lieb algebra, Geom. Topol. 13 (2009), 709-741.

[7] P. Fendley and V. Krushkal, Link invariants, the chromatic polynomial and the Potts model, Adv. Theor. Math. Phys. 14 (2010) 2, 507-540.

[8] D.W. Hall, On golden identities for constrained chromials, J. Combinatorial Theory Ser. B 11 (1971), 287-298.

[9] F. Jaeger, On the Penrose number of cubic diagrams, Graph colouring and variations. Discrete Math. 74 (1989), 85-97.

[10] V.F.R. Jones, Subfactors and knots. CBMS Regional Conference Series in Mathematics, 80. Published for the Conference Board of the Mathematical Sciences, Washington, DC; American Mathematical Society, Providence, RI, 1991.

[11] L.H. Kauffman and P. Vogel, Link polynomials and a graphical calculus, J. Knot Theory Ramifications 1 (1992), 59-104.

[12] P. Martin and D. Woodcock, The partition algebras and a new deformation of the Schur algebras. J. Algebra 203 (1998), no. 1, 91-124.

[13] S. Morrison, E. Peters and N. Snyder, Knot polynomial identities and quantum group coincidences, Quantum Topol. 2 (2011), 101-156.

[14] R. Penrose, Applications of negative dimensional tensors, 1971 Combinatorial Mathematics and its Applications (Proc. Conf., Oxford, 1969) pp. 221-244. Academic Press, London.

[15] N.Yu. Reshetikhin and V.G. Turaev, Ribbon graphs and their invariants derived from quantum groups, Comm. Math. Phys. 127 (1990), 1-26.

[16] D. Treumann, How many chromatic polynomials of planar maps are there?, https://mathoverflow.net/q/241734

[17] D. Treumann and E. Zaslow, Cubic Planar Graphs and Legendrian Surface Theory, arXiv:1609.04892

[18] W.T. Tutte, A census of planar maps, Canad. J. Math. 151963 249-271.

[19] W.T. Tutte, On chromatic polynomials and the golden ratio, J. Combinatorial Theory 9 (1970), 289-296.

[20] W.T. Tutte, More about chromatic polynomials and the golden ratio, Combinatorial Structures and their Applications, 439-453 (Proc. Calgary Internat. Conf., Calgary, Alta., 1969)

[21] W.T. Tutte, A contribution to the theory of chromatic polynomials, Canadian J. Math. 6, (1954), 80-91.

[22] K. Walker, Deletion-contraction relations, hard hexagons, and the shadow world, Talk at IPAM, 2007. Available at http://canyon23.net/math/talks/

[23] H.Wenzl, On a sequence of projections, C. R. Math. Rep. Acad. Sci. Canada 9 (1987), 5-9.

[24] S. Yamada, An invariant of spatial graphs, J. Graph Theory 13 (1989), 537- 551. 
IAN AGOL

University of California, Berkeley, 970 Evans Hall \#3840, Berkeley, CA, 947203840

E-mail address: ianagol@berkeley.edu

VYACHESLAV KRUSHKAL

Department of Mathematics, University of Virginia, Charlottesville, VA 22904-4137 USA

E-mail address: krushkal@virginia.edu 